12

\title{
Ионно-оптическая система источника ионов с фокусировкой по энергии в формируемом пучке
}

\author{
(c) И.А. Аверин, ${ }^{1}$ А.С. Бердников, ${ }^{1}$ С.В. Масюкевич, ${ }^{1}$ Н.С. Самсонова, ${ }^{2}$ Н.Р. Галль, ${ }^{2}$ Л.Н. Галль ${ }^{1}$ \\ ${ }^{1}$ Институт аналитического приборостроения РАН, \\ 190103 Санкт-Петербург, Россия \\ ${ }^{2}$ Физико-технический институт им. А.Ф.Иофрфе РАН, \\ 194021 Санкт-Петербург, Россия \\ e-mail: agreer@bk.ru
}

(Поступило в Редакцию 5 июня 2018 г.)

Рассмотрены пути снижения потерь интенсивности ионного пучка в масс-спектрометрическом источнике ионов, вызванные хроматической аберрацией его иммерсионной ионно-оптической системы. Эти потери весьма значимы при формировании пучков из ионов с большим разбросом по энергии, и уменьшение аберрационных потерь особенно актуально при использовании источников ионов совместно с массанализаторами с фокусировкой по энергии. Показано, что эти потери могут быть существенно снижены при использовании нового типа ионно-оптической системы источника, включающей в себя ахроматические элементы. Приведен специальный метод расчета таких элементов. Компьютерные моделирования показали высокую эффективность подобных элементов в ионно-оптическом тракте источников ионов массспектрометров.

DOI: $10.21883 /$ JTF.2019.04.47321.222-18

\section{Введение}

Успехи, достигнутые масс-спектрометрией в последние годы, связаны в первую очередь с освоением большого числа новых методов ионизации веществ, большинство которых характеризуется значительным разбросом по энергии образующихся ионов. Это требует особого внимания к формированию ионных пакетов и ионных пучков перед их разделением по массе во времяпролетных и статических масс-анализаторах $[1,2]$.

Времяпролетные масс-спектрометры незаменимы при получении разнообразной информации о молекулах в биохимических исследованиях, но плохая форма регистрируемого пика вблизи его основания ограничивает их применение в изотопном и элементном анализе. Для этих типов анализа по-прежнему наиболее распространенными остаются статические магнитные масс-спектрометры с одинарной и двойной фокусировкой, обеспечивающие разделение пиков масс, различающихся по амплитуде на пять порядков и более [3]. Важнейшим условием получения для статических масс-спектрометров высокой разрешающей способности в таком динамическом диапазоне, наряду с низким уровнем аберрационного уширения, обеспечиваемого масс-анализатором, являются геометрические и энергетические параметры пучка ионов, формируемого источником.

С электронно-оптической точки зрения масс-спектрометрический источник ионов представляет собой эмиссионный объектив с объемным и неустойчивым по форме и местоположению „катодом“, что делает весьма сложной задачу формирования стабильного двумерного пучка ионов с эмиттансом, удовлетворяющим условию его максимального согласования с аксептансом магнитного масс-анализатора [4]. Этому условию для магнитных анализаторов с одинарной фокусировкой, как правило, отвечают пучки ионов с достаточно разумными геометрическими характеристиками (отношением ширины к высоте пучка не менее чем $1: 100$, углами расходимости порядка сотых радиана) и разбросом по энергии, не превышающим $10^{-4}$ от полной энергии. Одновременное выполнение этих требований является сложнейшей ионно-оптической задачей, над решением которой разработчики статических масс-спектрометров бьются уже не менее 100 лет и которая тем не менее остается проблемной и до настоящего времени. В сложившейся ионно-оптической практике ее решают при помощи иммерсионных фокусирующих систем, состоящих из последовательно расположенных диафрагм [5]. Расчет этих систем проводится с помощью специальных программ, традиционные электронно-оптические подходы, основанные на расчетах линз в параксиальном приближении, уже давно не используются. Правильное проектирование таких систем сопряжено со значительными вычислительными трудностями, связанными с формированием пучка из множества ионов, ограниченного контуром первичного эмиттанса большого объема с нестабильными границами.

Для источников ионов, в которых ионы образуются с малым разбросом по энергии, не превышающем $0.1 \%$ от их средней энергии, эта задача в значительной степени решена: иммерсионная ионно-оптическая система источника ионов за счет ускорения ионов не менее чем на два порядка и умеренной коллимации пучка обеспечивает геометрические параметры выходного эмиттанса ионов, позволяющие вписать его эмиттанс в 


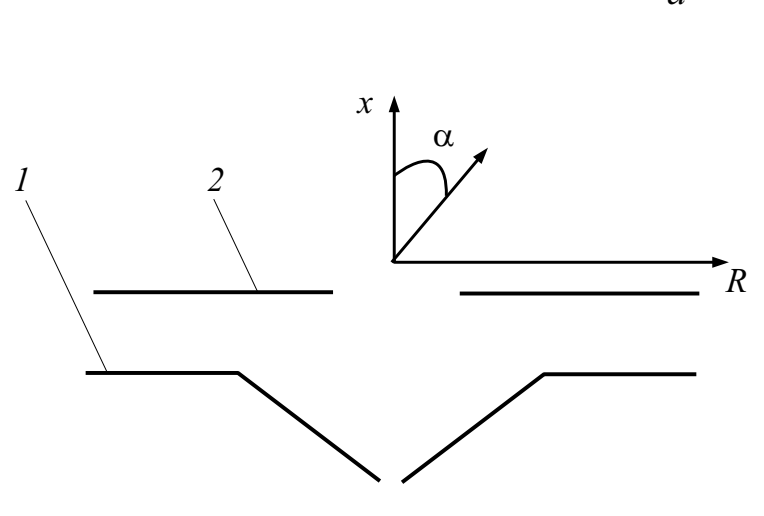

$b$

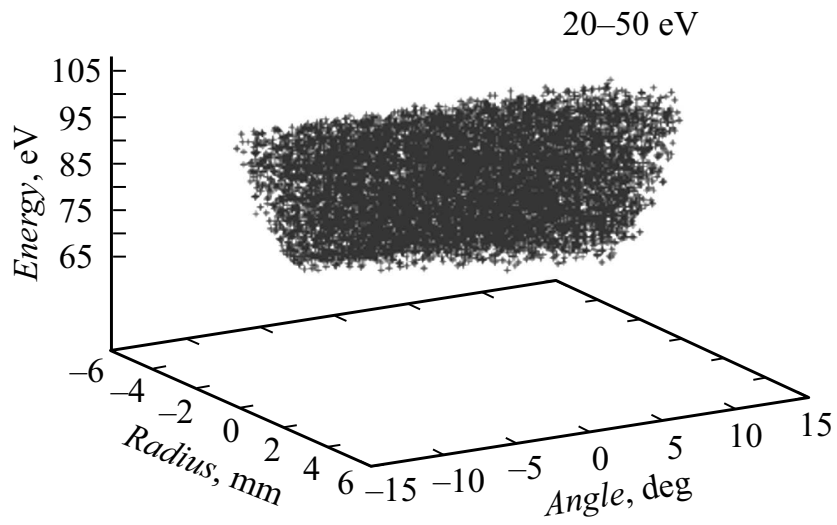

Рис. 1. $a-$ выходной узел интерфейса источника ионов с электрораспылением и $b$ - типичный для метода „электроспрей“ эмиттанс ионов, входящих в ионно-оптическую систему, формирующую ионный пучок. 1 - скиммер интерфейса, $2-$ выходная диафрагма - плоскость регистрации входного эмиттанса ионного пучка, $R-$ радиус пучка, $\alpha-$ угол расходимости, $E-$ распределение по энергии образовавшихся ионов.

аксептанс масс-анализатора при минимальных потерях общей интенсивности.

Для источников ионов с новыми методами ионизации задача формирования ионного пучка для статического масс-спектрометра оказывается значительно более сложной, поскольку первичный эмиттанс ионов в этом случае имеет энергетический размер, составляющий приблизительно $1 \%$ от конечной энергии пучка, что при формировании ионного пучка в стандартном источнике ионов приводит к потере интенсивности не менее чем на порядок. На рис. 1 представлен типичный эмиттанс ионного пучка в координатах размер-угол-энергия на выходе из интерфейса источника ионов с электрораспылением (электроспреем).

Как видно из рисунка, первичный поток ионов, входящий в ионно-оптическую систему источника ионов из отверстия диафрагмы, имеет в среднем не только заметную энергию и доходящий до $50-70 \%$ ее разброс, но и большой угол расходимости на выходе из диафрагмы 2 , составляющий приблизительно $\pm 15^{\circ}$.

Для борьбы с разбросом по энергии в современных масс-спектрометрах часто устанавливают дополнительный элемент, называемый „ионным гидом“, внутри которого ионный пучок теряет энергию в столкновениях с нейтральными молекулами газа [6]. Однако метод дорог, он требует установки дополнительной ступени откачки, и хорошо работает только для достаточно тяжелых ионов, а потому применяется в основном в биохимической масс-спектрометрии. Что же касается ионов легких элементов, то они в ионном гиде не термализуются, а только рассеиваются на большие углы и теряются, в результате чего их потери только возрастают [7]. Как показали проведенные исследования, устранить потери интенсивности ионного пучка в формирующей ионно-оптической системе источника ионов возможно, введя в нее дополнительную ахроматическую фокусирующую линзу.

\section{Теоретические подходы к ахроматической фокусировке пучков заряженных частиц}

Создание ионно-оптической системы для формирования ионного пучка, эмиттанс которого будет в достаточной мере совмещен с аксептансом статического масс-анализатора из потока частиц с большими угловым, линейным и энергетическим разбросами, простым компьютерным перебором линзовых систем заведомо невозможно. Решение такой задачи требует применения аналитических методов, позволяющих сформулировать постановку задачи в общем виде. Такая задача, поставленная и решенная в [8], в математической постановке выглядит следующим образом.

Пусть имеется некоторый объемный источник заряженных частиц и пусть на некоторой поверхности вблизи него с криволинейными координатами $\xi, \eta$ плотность $J$ потока частиц $N$ в направлении движения пучка с углами $\theta, \gamma$ при энергии $E$ для заряда $q$ и массы $m$ с учетом возможной зависимости от времени $t$ имеет вид $N=N(\xi, \eta, \Theta, \gamma, E, q, m, t)$. Эту поверхность далее будем рассматривать как эквивалентный поверхностный эмиттер, и задача состоит в том, чтобы найти электростатическое поле, преобразующее распределение $N$ в новое распределение на $M\left(\xi^{*}, \eta^{*}, \theta^{*}, \gamma^{*}, E^{*}, q^{*}, m^{*}, t^{*}\right)$ поверхности $K$, рассматриваемой далее как источник для масс-анализатора. При этом само преобразующее поле не должно влиять ни на работу источника ионов, ни на работу масс-анализатора.

В работе [8] было показано, что существует класс электростатических полей, осуществляющих ахроматическую транспортировку пучка заряженных частиц, с гиперболическими электрическими потенциалами вида

$$
\Phi=\frac{a^{2} x^{2}}{2}+\frac{b^{2} y^{2}}{2}-\frac{\left(a^{2}+b^{2}\right) z^{2}}{2},
$$




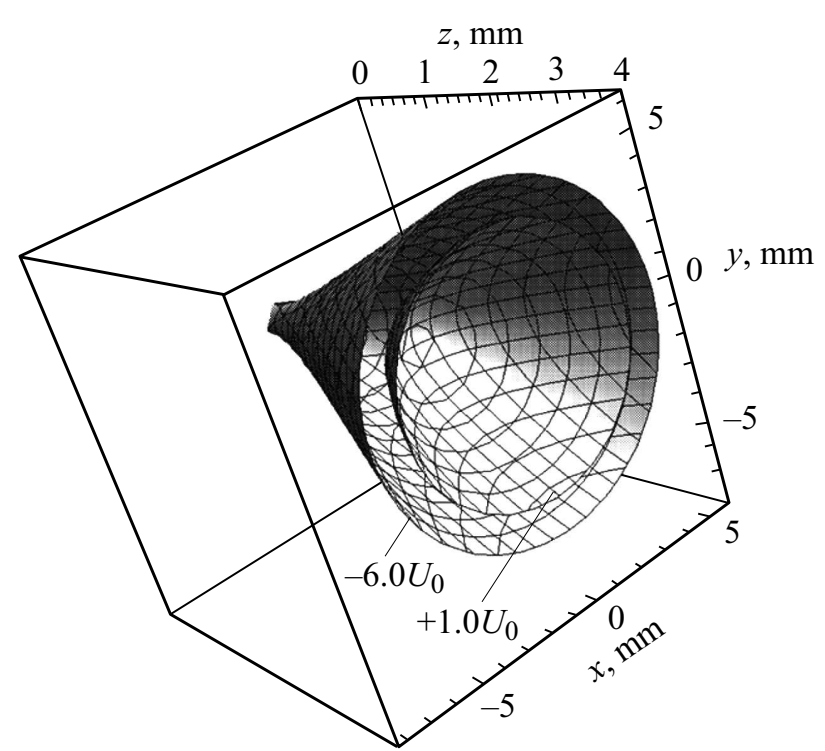

Рис. 2. Эквипотенциальные поверхности электрического потенциала $U(x, y, z)=\left(U_{0} / D^{2}\right)\left(\left(x^{2}+y^{2}\right) / 2-z^{2}\right)$, где внешняя поверхность соответствует значению $+1.0 U_{0}$, внутренняя поверхность - значению $-6.0 U_{0}, D-$ нормирующий множитель, $U_{0}-$ нормирующий множитель для потенциала.

эквипотенциальные поверхности которых являются однополостными и двухполостными гиперболоидами, при $\Phi=0$, вырождающимися в конус, причем в сечении их плоскостями, параллельными $O X Y$, получаются эллипсы с отношением полуосей $a / b$. В плоскости имеем квадратичное распределение электрического потенциала

$$
\varphi=\frac{a^{2} x^{2}+b^{2} y^{2}}{2},
$$

так что уравнения движения вдоль осей $O X$ и $O Y$ являются линейными и имеют вид

$$
\ddot{x}=-\frac{q a^{2}}{m} x, \quad \ddot{y}=-\frac{q b^{2}}{m} y .
$$

Интегралы этих уравнений соответствуют ограниченным колебаниям, и легко показать, что при $b=n a$ (где $n-$ натуральное число) в момент времени $t=(\pi / a) \sqrt{m / q}$ для всех частиц пучка имеет место идеальная фокусировка независимо от их энергии и угла вылета. Подбирая $n$, можно строить идеально вытянутые по оси $x$ эллиптические гиперболоиды, удобные для практики.

Не менее интересным для практики является поле вида

$$
\varphi=\frac{a^{2} x^{2}}{2}+p(y),
$$

где $p(y)$ - произвольная однозначная аналитическая функция. Для этого поля в [8] доказана теорема, утверждающая, что в полях типа (4) всегда существует бездисперсионная фокусировка второго порядка по углу при надлежащем выборе позиции приемника относительно источника. Эквипотенциальные поверхности такого поля - осесимметричные гиперболоиды, представленные на рис. 2. Физической основой такого утверждения является очевидная конфигурация поля, в которой во всей области движения частиц при отклонении от плоскости $z=0$ возникает сила, возвращающая частицу назад.

При реализации свойств поля (4) в реальной ионнооптической системе для создания ахроматической линзы возникает сложная задача его аппроксимации набором эквипотенциальных электродов простых форм, удобных на практике и сохраняющих основные ионно-оптические характеристики поля. Для источника ионов задача аппроксимации существенно упрощается по сравнению с аппроксимациями для энергоанализирующих систем, поскольку удовлетворительным будет уже такое техническое решение, при котором создается хотя бы возврат к плоскости $z=$ const частиц с сильно различающимися энергиями без требований формирования изображения источника.

\section{Аппроксимация ахроматического поля и численное моделирование свойств ионной оптики для источника ионов с большим разбросом по энергии}

На рис. 3 представлена ионно-оптическая система источника ионов, нацеленная на фокусировку потока ионов, выходящего из системы скиммер-диафрагма. Система не включает в себя цилиндрических линз (диафрагм) и состоит из двух главных элементов: иммерсионной линзы, состоящей из двух цилиндров с равными диаметрами, осуществляющей доускорение первичного пучка и его фокусировку по углу расходимости на отверстие в электроде 5, и последующей ахроматической линзы, полученной аппроксимацией двух эквипотенци-

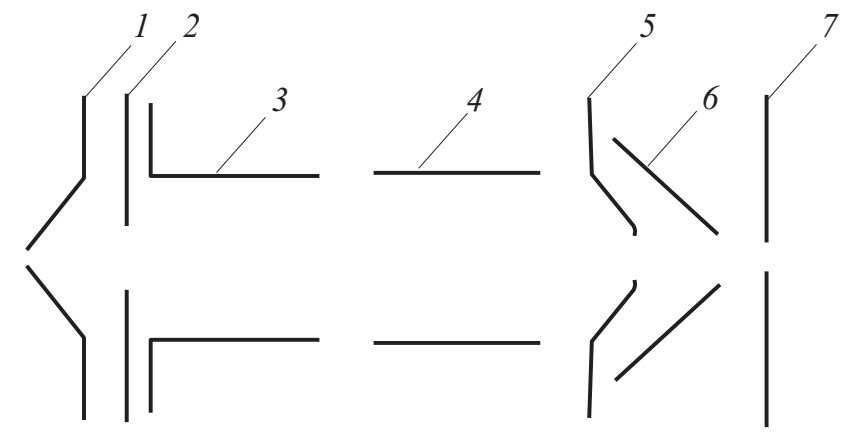

Рис. 3. Схема источника ионов с ахроматичной ионнооптической системой: 1 - скиммер источника ионов, $2-$ диафрагма постинтерфейсного пространства, 3 - первый электрод иммерсионной линзы, $4-$ второй электрод иммерсионной линзы, 5,6- входной и выходной электроды ахроматической системы, 7 - выходной электрод ионнооптической системы источника ионов. 


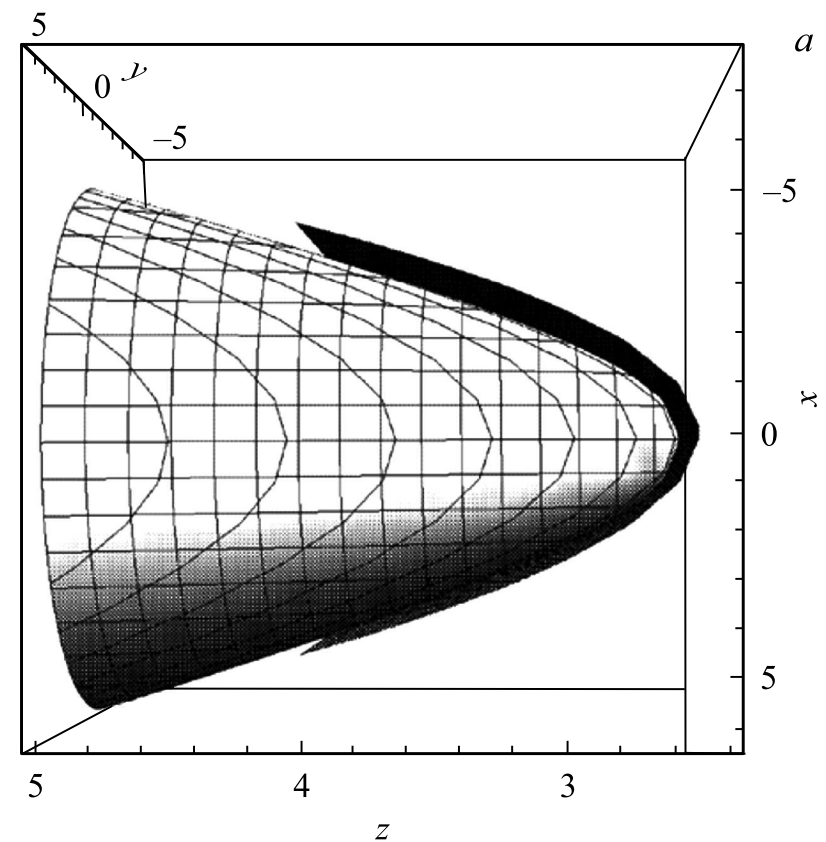

$a$

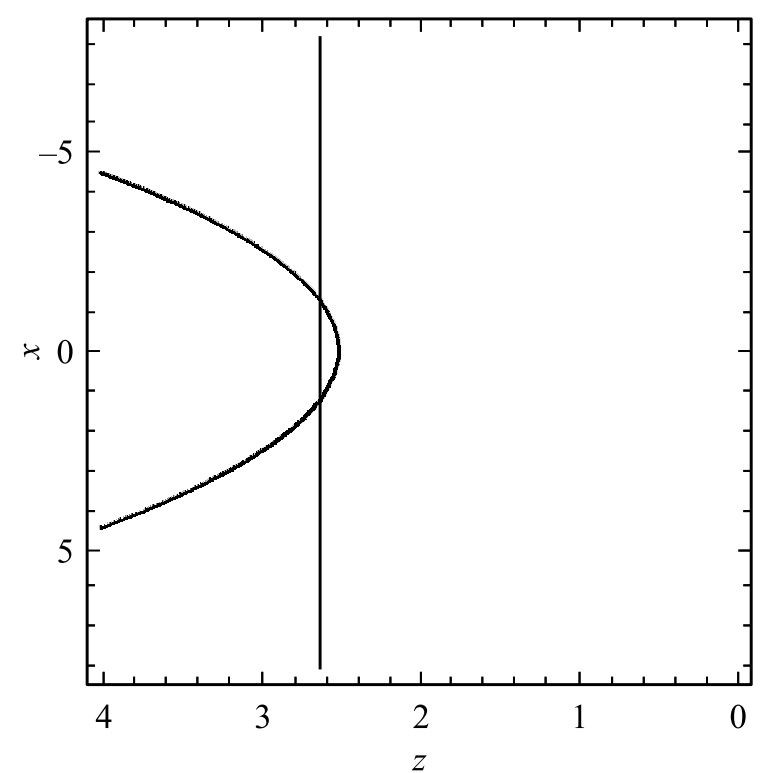

Рис. 4. Аппроксимация входного электрода ахроматической линзы: $a$ - парабола, фигура вращения которой аппроксимирует входной электрод ахроматической системы; $b$ - линия среза, которая позволяет получить входную щель в электроде.

альных поверхностей поля

$$
U(x, y, z)=\frac{U_{0}}{D^{2}}\left(\frac{1}{2}\left(x^{2}+y^{2}\right)-z^{2}\right),
$$

где $D-$ нормирующий множитель, имеющий смысл характерного расстояния, которое является частным случаем поля (1) при $a=b=1$. При этом входной электрод 2 ахроматической системы является выходным электродом иммерсионной линзы. Ахроматическая линза, составленная из электродов 5, 6, фокусирует ионный пучок с большим разбросом по энергии в условиях его торможения. Оптимизация размеров и потенциалов ионно-оптической системы источника ионов в целом состоит в таком их подборе, чтобы на выходе ионнооптической системы источника ионов был сформирован эмиттанс ионного пучка, наилучшим образом вписанный в аксептанс масс-анализатора. Выходной электрод 7 системы является диафрагмой с выходной щелью источника ионов и находится под потенциалом электрода 5, задавая энергию ионного пучка. Электроды 5 и 6 получаются аппроксимацией эквипотенциальных поверхностей поля (5), представленных на рис. 2.

В этой системе иммерсионная линза 2-5 осуществляет ускорение ионного пучка до его полной энергии, получаемой ионами при прохождении электрода 5 . Ахроматическая система из электродов 5-7 является одиночной линзой с торможением пучка в поле между электродами 5 и 6 . Электроды 5 и 6 получены аппроксимацией эквипотенциальных поверхностей, представленных на рис. 2: электрод $5-$ аппроксимацией эквипотенциальной поверхности, которая может

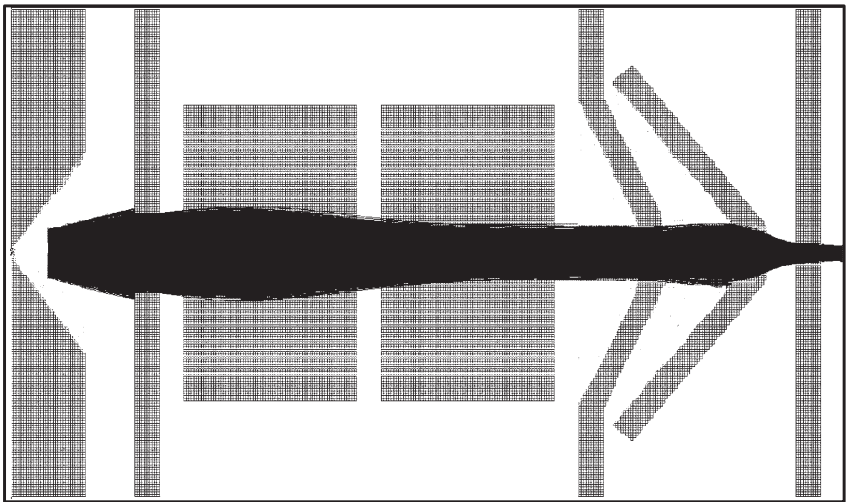

Рис. 5. Траектории пучка заряженных частиц в ионнооптической системе (плоскость $X Y$ ).

быть аппроксимирована с хорошей точностью параболой (рис. 4, $a$ ), а электрод 6 - эквипотенциальной поверхности . Вершины обеих фигур аппроксимации срезаются для обеспечения прохождения ионного пучка. Оптимальной является линия среза (рис. 4,b). Электрод 6 представляет собой конус с полууглом раствора $50.2^{\circ}$ и длиной электрода с внешней стороны $8.5 \mathrm{~mm}$. Электрод 7 ахроматической системы является выходным электродом оптики источника ионов в целом.

Численное моделирование проводилось в программе SIMION [9]. Первичным источником ионов для ахроматической системы является система скиммер-диафрагма, к которой была приложена разность потенциалов $50 \mathrm{~V}$. Эмиттанс ионного пучка на выходе из диафрагмы представлен на рис. $1, b$. Разброс по энергии во входном 

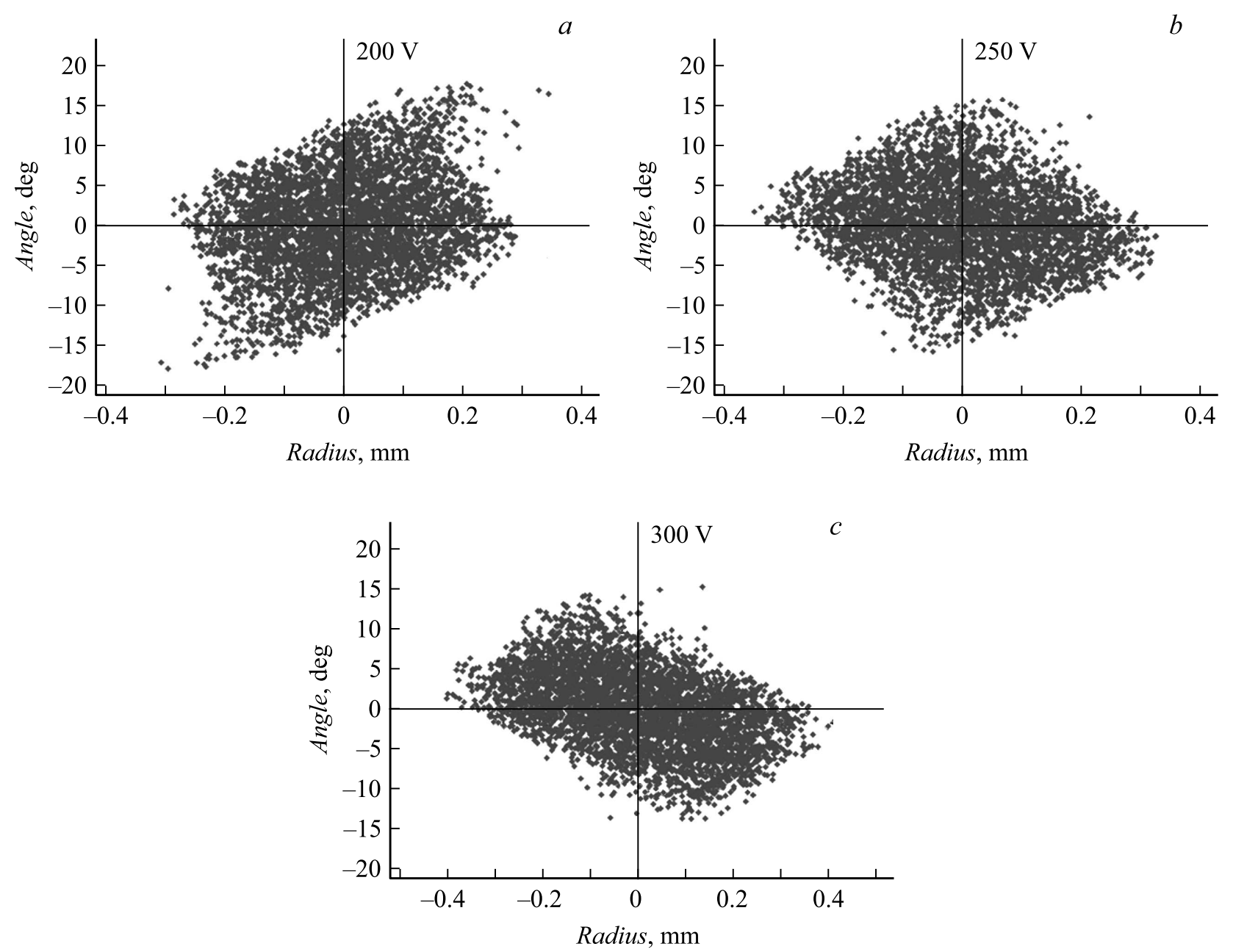

Рис. 6. Эмиттансы на выходе из ионно-оптической системы при разных значениях напряжения на управляющем электроде 6: $a-200, b-250, c-300 \mathrm{~V}$.

ионном пучке составляет $\pm 45 \%$. При моделировании разброс по энергии учитывался путем расчета пяти пучков с энергиями 20, 30, 35, 40, $50 \mathrm{eV}$ по 1000 ионов в каждом.

В результате проведенного численного эксперимента и последующей оптимизации были найдены режимы, обеспечивающие одновременную фокусировку 90\% первичного ионного пучка по углу и по энергии для условий, когда разброс по энергии на выходе из источника ионов составляет не менее $10^{-2}\left(e U_{a c c}\right)$, где $U_{a c c}-$ ускоряющее напряжение, $e-$ заряд электрона. Траектории ионов в области ионной оптики представлены на рис. 5.

Численное моделирование показало, что ахроматическая линза обладает очень сильным фокусирующим действием, способным кардинально менять параметры ионного пучка сформированного ионной оптикой источника. На рис. 6 представлены эмиттансы ионного пучка с разбросом энергии до $1 \%$ относительно ускоряющего напряжения и $\pm 45 \%$ относительно входного ионного пучка, показывающие его изменение от расходящегося к сходящемуся при изменении управляющего напряжения $U_{\text {contr }}$ на электроде 6 всего лишь в диапазоне от 200 до $300 \mathrm{~V}$.

\section{Вывод}

Синтезирована иммерсионная ионно-оптическая система нового типа, не имеющая в своем составе щелевых линз-диафрагм и с минимальными потерями, обеспечивающая передачу пучка ионов с большим исходным разбросом по энергии от источника ионов на вход массанализатора. Она включает ахроматическую линзовую систему, основанную на электростатическом поле с квадратичным электрическим потенциалом $\propto-z^{2}$, которая позволяет эффективно фокусировать ионные пучки с высоким разбросом ионов по энергии. Использование такой системы позволяет существенно повышать чувствительность масс-спектрометрических исследований на основе методов ионизации, принципиально связанных с получением ионов с большим разбросом по энергии, включая ВИМС, разрядные и газодинамические методы. 
Настоящая работа в части вклада авторов Аверин И.А., Бердников А.С., Масюкевич С.В., Галль Л.Н. выполнена в рамках гос. задания № 007-00229-18-00.

Авторы выражают свою благодарность К.В. Соловьеву и Н.К. Красновой (кафедра физической электроники Санкт-Петербургского политехнического университета Петра Великого) за полезные консультации.

\section{Список литературы}

[1] Галль Л.Н. Физические основы масс-спектрометрии и ее применение в аналитике и биофизике. СПб.: Изд-во политехн. ун-та, 2010. 164 с.

[2] Yavor M.I. Optics of Charged Particle Analyzers. Elsevier, 2009. $373 \mathrm{p}$.

[3] Галль Л.Н., Бажкенов А.Н., Кузьмин А.Г., Галль Н.Р. Сравнительные возможности масс-анализаторов разных типов в решении аналитических задач масс-спектрометрическими методами. // Масс-спектрометрия. 2008. Т. 5. № 4. C. $295-300$.

[4] Бердников А.С., Галль Л.Н., Хасин Ю.И. // Научное приборостроение. 2001. Т. 11. № 4. С. 28-34.

[5] Бердников А.С., Галль Л.Н., Саченко В.Д., Хасин Ю.И., Сапрыгин А.В., Калашников В.А., Залесов Ю.Н., Малеев А.Б. // Научное приборостроение. 2003. Т. 13. № 4. C. $3-21$.

[6] Ruotolo B.T., Benesch J.L.P., Sandercock A.M., Hyung S.-J., Robinson C.V. // Nature Protocols. 2008. Vol. 3. P. 1139-1152.

[7] Krutchinsky A.N., Padovan J.C., Cohen H., Chait B.T. // J. Am. Soc. Mass Spectrom. 2015. Vol. 26. N 4. P. 649-658.

[8] Галль Л.Н., Голиков Ю.К. // Научное приборостроение. 2014. T. 24. № 1. C. 11-17.

[9] SIMION: Ion and Electron Optics Simulator URL: http://simion.com 